\title{
An overview of jets and outflows in stellar mass black holes
}

\author{
Rob Fender • Elena Gallo
}

Received: date / Accepted: date

\begin{abstract}
In this book chapter, we will briefly review the current empirical understanding of the relation between accretion state and and outflows in accreting stellar mass black holes. The focus will be on the empirical connections between X-ray states and relativistic ('radio') jets, although we are now also able to draw accretion disc winds into the picture in a systematic way. We will furthermore consider the latest attempts to measure/order jet power, and to compare it to other (potentially) measurable quantities, most importantly black hole spin.
\end{abstract}

Keywords Black hole physics $\cdot$ X-ray binaries · Jets

\section{Introduction}

Jets, collimated relativistic outflows carrying large amounts of energy away from the deepest parts of the gravitational potential well, are a relative latecomer to the overall picture of accretion around stellar mass black holes. Much of the basics of accretion theory, namely how the matter gets in while angular momentum and radiation get out, was developed in between the 1960s and 1980s, with roots in much earlier works, and is still applicable today (and much of it will have been covered in other chapters in this book). In contrast, it took until the 2000s for it be accepted that jets are a key part of the accretion process in stellar mass accretors, both in terms of their uniquity and importance in having very large kinetic powers. Furthermore, despite knowledge of their existence in active galactic nuclei for a century, the basics of how relativistic jets are launched are still a long way behind our understanding of the accretion process.

R. P. Fender

Astrophysics, Department of Physics, University of Oxford, Keble Road, OX1 3RH, Oxford, UK

E-mail: rob.fender@astro.ox.ac.uk

E. Gallo

Department of Astronomy, University of Michigan, 500 Church St., Ann Arbor, MI 48109, USA 


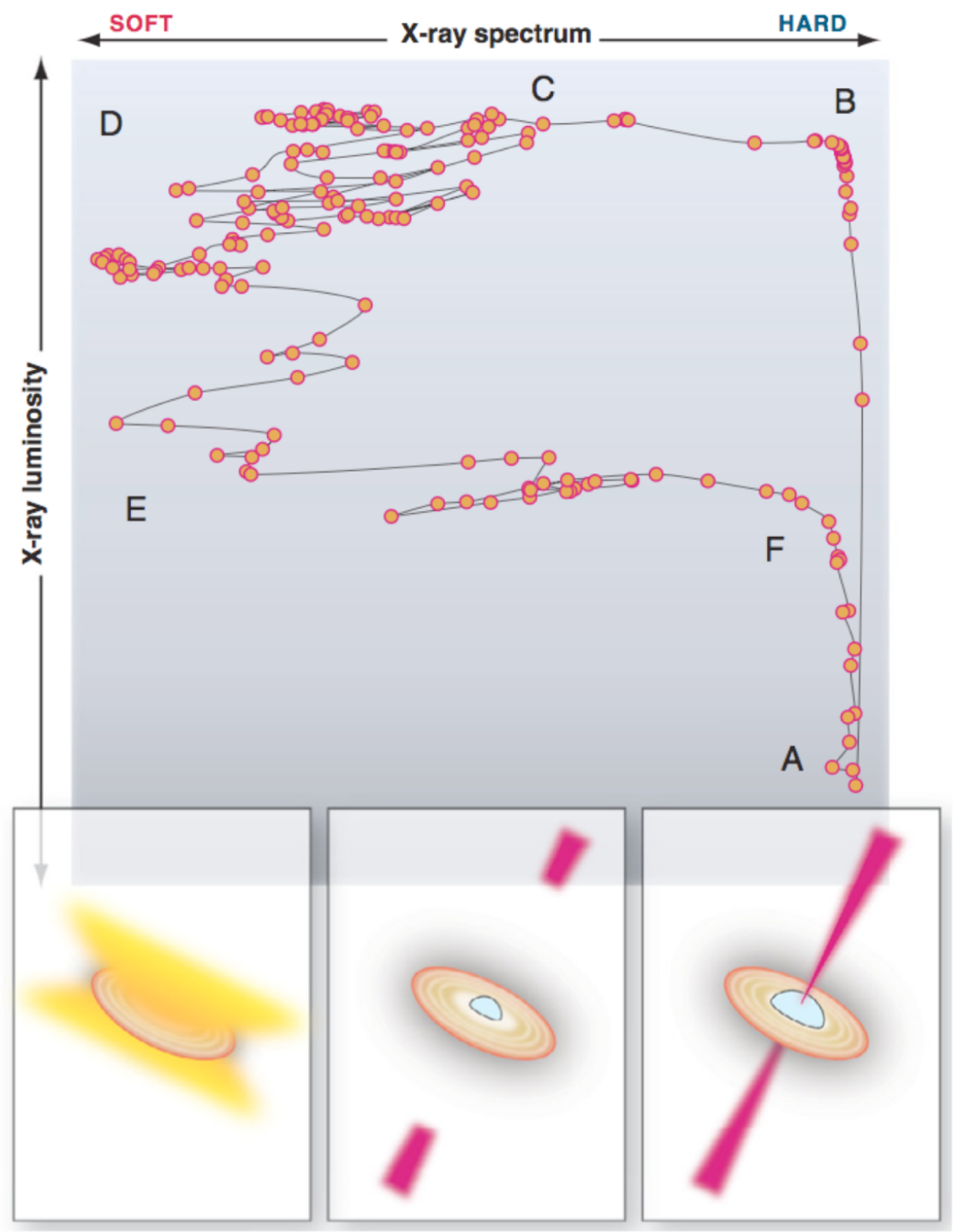

Fig. 1 A qualitative summary of the relation between accretion states and outflows in black hole X-ray binaries. The upper panel represents the relation between X-ray spectral hardness and luminosity, and the path A-B-C-D-E-F represents the path taken by the recurrentlyoutbursting binary GX 339-4 over the course of about one year. Hard states (to the right) are well-studied and show quasi-steady radio jets. Soft states (to the left) appear to have no radio jets but strong accretion disc winds. Transitions between the states, at least at high luminosity (e.g. B-C-D), appear to be associated with discrete jet ejection events. From Fender \& Belloni (2012), where a concise summary of the cycle can be found. 
Nevertheless, once we discovered that jets in X-ray binaries follow certain patterns, revealed mainly (but not exclusively) by coordinated observing campaigns in the radio and X-ray bands, we realised that they offered perhaps our best opportunity to understand the connection between accretion and jet formation in relativistic objects.

In this paper we will describe the current state of play in our empirical understanding of the relation between accretion, jets and winds in stellar mass black holes (and neutron stars). We will focus on thoroughly summarising the observational evidence, and highlighting recent progress.

For a lengthier introduction to the basic observables of jets and what can be understood from them, the reader is directed towards Fender (2006) and references therein. For a very quick summary of the state of the field, see Fender \& Belloni (2012). Other relevant historical reviews include Hjellming \& Han (1995), Mirabel \& Rodríguez (1999), Martí (2005).

\section{The empirical picture}

In this section we shall summarise the empirical evidence for a connection between accretion and outflow in X-ray binaries. We shall start with the black hole X-ray binaries, for which the evidence is strongest. Fig 1 summarises both the current state of our knowledge of the connection between accretion and outflows in black hole X-ray binaries, and how we most commonly choose to represent it. This representation of the relation between jets and X-ray state originated with Fender, Belloni \& Gallo (2004), and has since been greatly augmented by the work of Ponti et al. (2012) who found a clear relation between accretion disc winds and the soft $\mathrm{X}$-ray state.

We are very fortunate that, on timescales fairly well tuned to $\mathrm{PhD}$ durations or grant cycles, we can observe a single stellar mass black hole in a binary system undergo accretion in a small number of different modes over a large range of luminosities. The cycle illustrated in Fig 1 takes place over about one year, as is illustrated in Fig 2, Noteworthy points to take from this figure are that the quasisteady jet is present most of the time, although mainly at low luminosities, that the transient/flaring jet phase constitutes a very small fraction of the outburst, and that for most of the time at the highest luminosities there appears to be no core jet but a strong disc wind.

The following subsections refer to the (quasi-cyclic) phases labelled A through $\mathrm{F}$ in Figs 1 and 2.

\subsection{A to $\mathrm{B}$ (and $\mathrm{F}$ to $\mathrm{A}$ ): quiescence to bright hard states}

Outbursts of black hole X-ray binaries (BHXRBs) seem to be caused by a switch in the viscosity of the accretion disc, associated with the ionisation of hydrogen. The most recent test of this theory, originally developed for white dwarf accretion in cataclysmic variables (Meyer \& Meyer-Hofmeister 1981), continues to support the scenario (Coriat, Fender \& Dubus 2012). Once the viscosity increases, the accretion rate at the inner edge of the accretion disc rises dramatically. Since this 


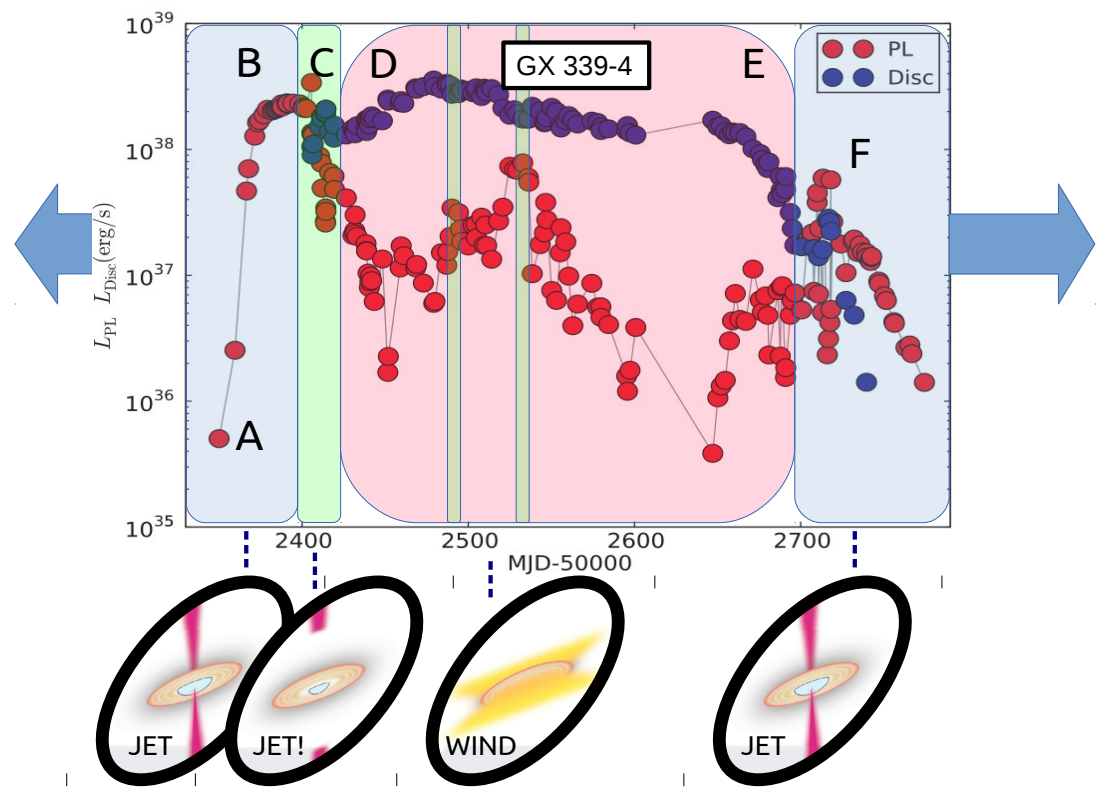

Fig. 2 An outburst of GX 339-4, this time shown as temporal evolution. The red and blue curves correspond to power-law and disc components of the X-ray spectrum: blue-dominated states are 'soft' and red are 'hard', in the context of Fig 1. The figure illustrates that the majority of the time at the highest luminosities is spent in the soft state where there appears to be non core radio jet, but instead a strong wind. The letters correspond approximately to those in Fig 1. Partially adapted from Dunn et al. (2010) and Fender \& Belloni (2012).

is where most of the accretion luminosity is produced, the source gets brighter on a short timescale (days).

In our experience to date, most BHXRBs spend most of their time outside of outbursts at 'quiescent' levels of accretion with corresponding luminosities of $\sim 10^{30-32} \mathrm{erg} \mathrm{s}^{-1}$. This population is not very well sampled, however, and some sources are stable at higher luminosities (e.g. V404 Cyg which is 'quiescent' at $\sim 10^{34} \mathrm{erg} \mathrm{s}^{-1}$ ) and selection effects suggests that there's likely to be a tail to lower luminosities. Once the outburst begins, however, the sources can rise to luminosities $\geq 10^{38} \mathrm{erg} \mathrm{s}^{-1}$ very rapidly, an extremely dramatic change. For most of this range in luminosity the X-ray spectrum remains in a 'hard' spectral state (however, it is likely that most of the action is going on in the ultraviolet, which is hard/impossible to observe in for most X-ray binaries, due to large dust extinction in the galactic plane). This state is characterised by a broad-band X-ray spectrum with photon index $\Gamma \sim 1.6-2.1$ (with the spectrum hardening as the luminosity increases; e.g. Plotkin et al. 2014) often observed to show a cutoff between $~ 50$ $130 \mathrm{keV}$ (with the temperature decreasing the luminosity increases; e.g., Motta et al 2009; Joinet et al. 2008), and generally ascribed to thermal Comptonisation in a hot plasma or 'corona' above/around/near the accretion disc. There is usually 
strong X-ray variability (up to $40 \%$ r.m.s. in the Fourier frequency range 0.01 $100 \mathrm{~Hz}$ ) associated with this state.

In this hard state, BHXRBs essentially always show relatively weak but steady radio emission, with a flat $\left(\alpha \sim 0\right.$, where flux density $\left.S_{\nu} \propto \nu^{\alpha}\right)$ spectrum and low levels of polarisation (Fender 2001; but see Brocksopp et al. 2013 and Russell $\&$ Shahbaz 2014). The relative steadiness, flat spectrum and lack of polarisation suggest that this emission originates in a more or less continuously replenished, partially self-absorbed outflow, such as those originally conceived of to explain the flat spectrum cores of some quasars. The radio-X-ray correlation has been studied extensively, and is discussed in more detail in the following subsection.

\subsubsection{Luminosity correlations}

Quasi-simultaneous radio and X-ray monitoring has become the standard tool of investigation for the so called "jet-accretion coupling" in hard state BHXRBs. A strong and repeating correlation has been established between the radio and X-ray luminosity for two systems, with $L_{\mathrm{X}}$ being proportional to $L_{\mathrm{r}}{ }^{0.6-0.7}$ (GX339-4: Corbel et al. 2003, 2013 and V404 Cyg: Gallo, Fender \& Pooley 2003; Gallo, Fender \& Hynes 2005, Corbel, Körding \& Kaaret 2008). However, the universality of this relation 10 years after its discovery is far from obvious. Fig 3 summarizes the current state of the problem by assembling what is likely the most complete data collection as of today (data from Gallo, Miller \& Fender 2012 plus Corbel et al. 2013, and references therein). Two main differences stand out with respect to the simple picture we used to draw a decade ago: firstly, there appears to be two luminosity tracks (see Gallo et al. 2012 for more details); secondly, the behavior of the BHXRB H1743-22 (in blue) as observed during the decline of its its 2008 outburst (Jonker et al. 2010; Miller-Jones et al. 2012; Miller et al. 2012) is in stark contrast with that of GX339-4 and V404 Cyg (green and red, respectively): H174322 starts off as under-luminous in the radio band during the initial outburst decay phase $\left(10^{36} \lesssim L_{\mathrm{X}} \lesssim 10^{38} \mathrm{erg} \mathrm{s}^{-1}\right)$, proceeds to make a nearly horizontal excursion toward lower X-ray luminosities (between $10^{36} \lesssim L_{\mathrm{X}}<\lesssim 10^{36} \mathrm{erg} \mathrm{s}^{-1}$ ), and finally reaches a comparable radio luminosity level (for the same $L_{\mathrm{X}}$ ) as GX339-4 and V404 Cyg (this happens below $\simeq 10^{34} \mathrm{erg} \mathrm{s}^{-1}$ ). Whatever drives the relative radio quietness/loudness of BHXRBs in hard and quiescent states, thus, cannot be linked to any physical property that remains constant over typical outburst time-scales (e.g., black hole spin, orbital inclination; see Soleri \& Fender 2011).

Coriat et al. (2011) ascribe the erratic behavior of H1743-22, and by analogy that of other radio-quiet systems, to the onset of a radiatively efficient inflow (see also Körding, Fender \& Migliari 2006). Along the same lines, a more theoretical basis for the existence of a radio-quiet (or rather, X-ray-bright) track has been recently proposed by Meyer-Hofmeister \& Meyer (2014), who argue that thermal photons from a weak, cool, inner disc (sustained by re-condensation of optically thin gas within the inner regions; Meyer et al. 2007; Liu et al. 2007) could be responsible for enhancing the seed photon field available for Comptonisation, and hence the hard X-ray flux. From a theoretical standpoint (as well as observational, see e.g. Miller et al. 2006; Reis Fabian \& Miller 2010; Reynolds \& Miller 2013 and references therein), the inner cold disc would cease to exist at low accretion rate. Thus, the radio-quiet track is not expected to extend down to the quiescent regime. Though this exact behavior is just what was observed in the case H17143-22, 
whether the inner disc re-condensation scenario will stand the test of time (in terms of reproducing the X-ray and radio behavior of BHXRBs) depends critically on our ability to probe the truly quiescent domain (see Calvelo et al. 2010 and MillerJones et al. 2011 for a detailed discussion on how distance limitations are likely to hamper a systematic investigation in the radio band). To date, A0620-00 is the only truly quiescent system with a robust radio detection, and its radio luminosity seems to lie on the extrapolation of the GX339-4 and V404 Cyg radio/X-ray correlations down to Eddington-scaled X-ray luminosities as low as $\sim 10^{-9}$ (Gallo et al. 2006). However, with a sample of one, and considering selection effects, this is a long way from conclusive.

At the same time, a systematic study on the radio properties (most notably spectral index and polarization degree) of BHXRBs while on the radio quiet/X-ray bright track could provide complementary information on the physics that drives this apparent divide (there are hints that the "radio quiet" objects have slightly more optically thin radio spectra, Brocksopp et al. 2013). A good candidate for the origin of the flat spectrum, left unresolved in Blandford \& Königl (1979), is internal shocks driven at the frequencies of the X-ray variability (Malzac 2013).

Determining the compact jet contribution at mm, infrared (IR) and optical frequencies is admittedly challenging; much progress has been made in this direction over the last 10 years. A growing number of works (based both on spectral energy distribution analysis as well as variability studies) support the claim that, while in the hard state, the compact jet extends all the way up to IR and often optical frequencies (Fender 2001; Chaty et al. 2003; Bradley et al. 2007; Gallo et al. 2007; Russell et al. 2010, 2011b; Brocksopp et al. 2010; Malzac et al. 2004; Hynes et al. 2004, 2006, 2009; Markoff \& Nowak 2007; Casella et al. 2010). Most relevant to this section, Russell et al. (2006) collected nearly-simultaneous IR/optical and Xray observations of $33 \mathrm{X}$-ray binaries (black holes and neutron stars) to estimate the relative contributions of various IR/optical emission processes as a function of X-ray luminosity. They found evidence for a positive correlation between the IR/optical and X-ray luminosity, of the form $L_{\mathrm{opt}-\mathrm{IR}} \propto{L_{\mathrm{r}}}^{0.6}$, extending all the way from the peak of the hard state down to the loses quiescent X-ray luminosities. Notwithstanding the large scatter, no strong outliers, exceptions, sub-clusters or bifurcation have been reported so far for this relation.

\subsection{B-C-D: Transition from hard to soft}

Once the rising source passes an X-ray luminosity of $\sim 10^{37} \mathrm{erg} \mathrm{s}^{-1}$ (albeit with substantial variations between sources and even between different outbursts of the same source; see, e.g., Dunn et al. 2010 ), at which level it will still be in the hard state, it is very likely to make a subsequent transition to a softer spectral state. The transition from the hard state to the soft state can occur with very little change (of order unity) in the broadband luminosity of the system (in spite of rather dramatic changes in the spectral shape), and has a number of very interesting characteristics. For a 'typical' BHXRB the spectral transition from hard to soft states can take days or weeks, but the evolution can be much more dramatic in terms of the X-ray power spectra. While the hard and soft states appear to be dominated by strong $(\geq 30 \%)$ and very weak $(\leq 5 \%)$ broad band noise respectively, 


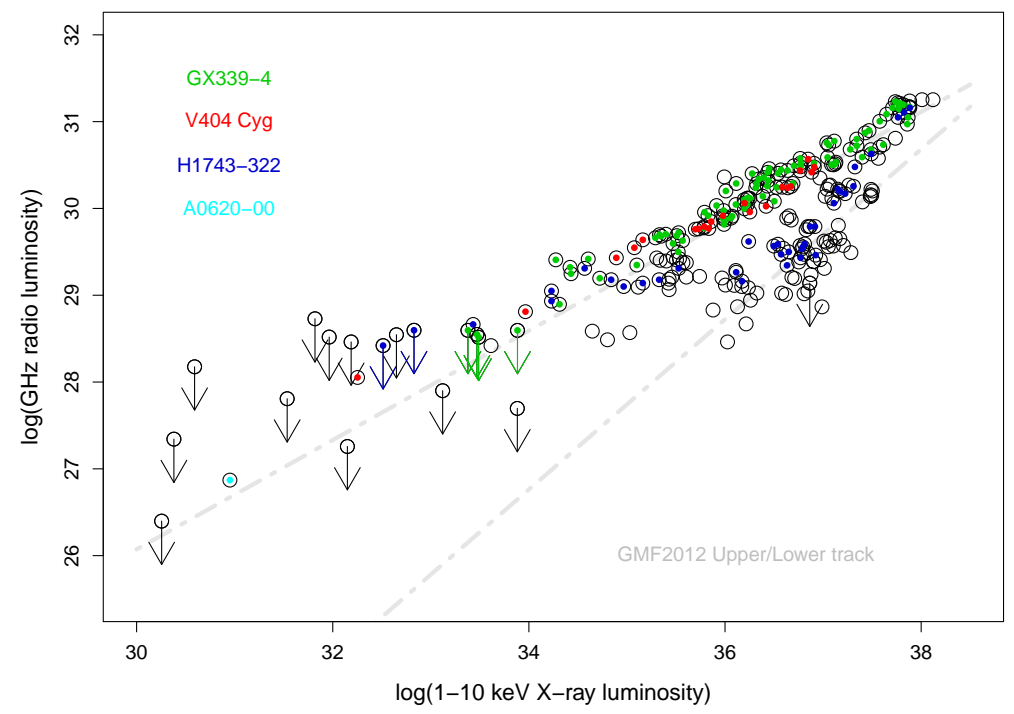

Fig. 3 The radio/X-ray luminosity plane of black hole X-ray binaries (c.a. 2014). Shown are quasi-simultaneous radio and X-ray luminosity measurements (in log CGS units) for 24 black hole X-ray binaries, ranging from quiescence (A0620-00; Gallo et al. 2006) up to bright hard states. The dashed grey lines indicate the best-fit relations to the upper and lower tracks as identified by Gallo et al. (2012) $\left(L_{\mathrm{r}} \propto L_{\mathrm{X}}{ }^{0.63}\right.$ and $L_{\mathrm{r}} \propto L_{\mathrm{X}}{ }^{0.98}$, respectively). We also highlight the two sources for which a tight non-linear correlation of the form $L_{\mathrm{X}} \propto L_{\mathrm{r}}{ }^{0.6-0.7}$ has been reported over a wide dynamic range (i.e. GX339-4; Corbel et al. 2003, and V404 Cyg; Gallo Fender Pooley 2003; Corbel, Körding \& Kaaret 2008), plus the enigmatic source H1743-322, which appeared to "jump" from the lower to the upper track as it faded into quiescence following its 2008 outburst (Jonker et al. 2010; Miller-Jones et al. 2012).

some phases during the transition can be completely dominated by strong peaked and QPO-like components. Casella et al. (2005) classified these QPOs into three types: A, B \& C, with strong type-Bs being the most indicative of the transition.

The hard to soft transition is also where the brighest radio flares occur, sometimes singly and sometimes in sequences. These radio flares can peak, in the $1-5$ $\mathrm{GHz}$ band, at many times the preceding hard state flux density, and (mostly) evolve in a 'standard' (e.g. van der Laan 1966) way from optically thick to thin as they (presumably) expand. Such flares are occasionally spatially resolved by radio telescopes into individual radio knots which move away from the radio core (presumably the site of the black hole) at (at least) mildly relativistic speeds (e.g. Mirabel \& Rodríguez 1994; Hjellming \& Rupen 1995; Fender et al. 1999; MIllerJones et al. 2012). It appears, although it is not at all proven, that these jets may move faster than those in the hard state, and so what we may be seeing is at least partially associated with internal shocks in a flow of variable (increasing) speed (Fender et al. 2004 and references therein; Fender et al. 2009). However, it is certainly not clear that they are significantly more powerful than the preceding, bright, hard state jets. 
There have been a number of attempts to associate the 'moment of jet launch' with an associated change or event in the accretion flow. Fender, Homan \& Belloni (2009) and Miller-Jones et al. (2012) both attempted to see if the momenet of jet launch could be associated with changes in the timing (X-ray variability) properties of a small number of black hole binaries, in particular with the occurrence of the type-B QPOs (see also discussion in Soleri, Belloni \& Casella 2008). While they are certainly broadly coincident, a one-to-one relation could not be firmly established and whether or not there exists a key signature of the moment of launch remains unclear. Seemingly at odds with this statement, in the bright black hole binary GRS 1915+105, which undergoes long cycles of accretion state changes on timescales as short as minutes, it is clear that each cycle is associated with an ejection event, and possibly even with a particular moment in that cycle (e.g. Mirabel et al. 1998; Klein-Wolt et al. 2001). However, these state changes appear to be compressed versions of what happens on longer timescales in other systems, and the blurring effect caused by the size scale of the radio emission makes direct association with some change difficult. Success is more likely in the infrared band, where much effort was made for GRS 1915+105 (e.g. Fender et al. 1997; Eikenberry et al. 1998; Mirabel et al. 1998) but it is extremely hard to maintain minutes-resolution monitoring of a more normal black hole binary across its entire week-long transition phase. Finally, it should be noted that there may be no clear 'moment of jet launch' at all: internal shock models are showing promise in reproducing some of the radio properties of X-ray binaries (e.g. Jamil et al. 2010; Malzac 2013) and it may be that a rapid, but not instantaneous, change in jet properties (such as injection cycle time or speed) are enough to reproduce what we see as bright flares in the radio band.

\section{$2.3 \mathrm{D}$ to $\mathrm{E}:$ The soft state}

In the soft state the X-ray spectrum is dominated by a blackbody-like component which peaks around $1 \mathrm{keV}$, combined with a steeper and weaker power law. The origin of the thermal component is likely to be an optically thick accretion disc. The overall X-ray variability drops dramatically in this state (often below $5 \%$ ). The core radio, $\mathrm{mm}$ and near-infrared emission drop dramatically in the soft state (Fender et al. 1999; Russell et al. 2011a), the simplest interpretation of which is that the core jet has switched off.

A major breakthrough in understanding states appears to have arrived recently with Ponti et al. (2012) demonstrating that accretion disc winds, revealed in Xray spectra, appear to be uniquely observed in edge-on, soft-state BHXRBs (caveat relatively poor sampling of late-time soft states, and some remaining small uncertainty about how evolving ionisation could affect this conclusion). Thus it seems that in moving from the hard to the soft state, we leave a regime of strong, quasisteady jets and little, if any accretion disc wind and enter the converse regime, with strong winds and weak jets (see also Miller et al. 2006, 2008; Neilsen \& Lee 2009). These two regimes are probably not simply a rebalancing of the same outflow power, however, with the wind probably carrying less kinetic power but more mass than the hard state jet (detailed calculations of kinetic energy, mass and momentum flux in these two modes have yet to be carried out, however). 
2.4 X-ray states: other views and potential origins

The X-ray states described above have been done so from a viewpoint which is very much biased towards the outflows. Other very useful reviews, usually more focussed on the X-ray emission and what it tells us about the workings of the inner accretion flow, can be found in Remillard \& McClintock (2006), Done, Gierlinski \& Kubota (2007), Belloni, Motta \& Munoz-Daris (2011). The general pattern of hysteresis can also be viewed in other ways than the hardness-intensity diagram used in Fig 1. In particular Munoz-Darias, Motta \& Belloni (2011) and Plant et al. (2014) utilised rms-intensity and reflection-intensity diagrams respectively to show broadly the same patterns but shedding new light on the detailed evolution of the accretion flow (see also Belloni \& Stella, this book).

It should be noted that while the root cause of the outburst may be agreed upon as being the hydrogren ionisation instability, the origin of the accretion state cycles and associated spectral hysteresis is not at all clear. There are published models which may be testable in the future (e.g. Petrucci et al. 2008; Begelman \& Armitage 2014 and references therein), and very similar cycles of behaviour appear to be present in systems containing both neutron stars and white dwarfs (Körding et al. 2008b; Munoz-Darias et al. in prep).

There are good reasons to believe that the patterns of behaviour observed in stellar-mass black holes should be scalable to intermediate mass black holes and supermassive black holes in active galactic nuclei, in fact there is strong observational support for this view. Merloni, Heinz \& Di Matteo (2003) and Falcke Körding \& Markoff (2004) presented the first evidence for relatively neat scalings of X-ray/radio luminosities and black hole mass, across the whole black hole mass range. McHardy et al. (2006) and Körding et al. (2007) made similar analyses for timing frequencies. Körding, Jester \& Fender (2006) made an argument that the overall patterns of coupling between states and jets might be the same in AGN, although this connection has really yet to be established convincingly (see also Körding, this book).

\section{Jet power}

Radiative and mechanical feedback from relativistic jets is thought to play a key role in regulating the growth of galactic bulges and their nuclear super-massive black holes. Empirical scaling relations between the mass of super-massive black holes in nearby galaxies and large scale properties of their host bulges, such as the well know "black-hole-mass/stellar velocity dispersion" relation (see Kormendy \& Ho 2013 for a recent review) are interpreted as strong observational evidence for strong co-evolution. At the same time, state-of-the-art cosmological simulations (e.g. Khandai et al. 2014) that trace the assembly and merger history of galaxies and their nuclear black holes rely on some form of black hole- (plus supernova) -driven feedback in order to reproduce them. Nevertheless, even the most sophisticated models make use of over-simplified recipes for the relative efficiencies of accretion vs. jet power, and posit that the jet power, at least during the quasar phases, is set by the spin of the black hole. As we discuss in the following sections, BHXBRs can actually help shed light on some of these processes. 
3.1 Jet power and black hole spin

The holy grail for relativistic jet astrophysics is to establish the jet formation mechanism. More specifically, we would like to be able to identify observational signatures pointing unequivocally to models that rely solely on differential rotation (Blandford \& Payne 1982) vs. models that tap directly into the rotational energy of the black hole (Blandford \& Znajek 1977; BZ). While super-massive black holes in AGN offer the advantage of vast demographics, BHXRBs in soft states provide us with a more favorable environment to systematically employ X-ray spectral fitting techniques to measure the temperature, and thus the extent, of the inner accretion disc (see Miller 2007 and Reynolds 2013 for recent reviews, as well as Reynolds, and McClintock, Narayan \& Steiner, this book). This translates into an estimate of the black hole spin parameter $a$, which can then be compared against the jet power $P_{\mathrm{j}}$ in order to (dis)prove a relation of the form $P_{j} \propto a^{2}$, predicted by the BZ model (see Tchekhovskoy et al. 2010 for a full relativistic traetment).

How to measure $P_{\mathrm{j}}$ ? When it comes to BHXRBs, persistent, hard state, compact jets are a natural place to start. Unfortunately, that the face-value radio luminosity of compact jets is at best a poor indicator of jet power is apparent from a number of considerations; firstly, although compact jets seem to be persistently on during hard states, their flux density varies by orders of magnitude, with some non linear power of $L_{\mathrm{X}}$ (see section 2.1.1). In addition, it should be kept in mind that any inference of radio luminosity that is based on a single-frequency flux density measurement relies on assuming a specific spectral shape at lower frequencies (often taken as flat). Further, extrapolating the integrated radio luminosity to total (i.e. radiative plus kinetic) jet power relies on even more crucial assumptions: $i$ ) on the location of the optically-thin-to thick jet break (which is known to vary with overall luminosity, yet not necessarily as basic scaling relations would predict; see next section); ii) on the location of the cooling break (possibly constrained in one source, see again next section) $i i i$ ) on the radiative efficiency of the synchrotron process.

Based on the above arguments, in attempt to test the presence of a correlation between jet power and spin parameter in hard state BHXRBs, Fender, Gallo \& Russell (2010) adopted a phenomenological approach whereby the relative normalization of the (thought to be universal) radio/X-ray and/or infrared/X-ray correlation were taken as a proxy for total jet power. Perhaps not surprisingly, no evidence for a positive correlation between these normalisations and reported spin measurements emerged - a result that could be interpreted as due the large uncertainties in measuring jet power (and, to a second extent, spin). A different approach was taken by King et al. (2013a), who found a marginally significant positive correlation between the mass-scaled radio luminosity and spin parameter across a sample of 11 BHRBs and 37 Seyfert galaxies.

Ideally, one would like to calibrate the radio luminosity-to-total jet power relation conversion via robust observational constraints on the amount of work exerted by the jets on the surrounding inter-stellar medium, and use jet-inflated radio lobes and cavities as effective jet calorimeters, similarly to what has been done for super-massive black holes in radio galaxies for decades. Unfortunately, the number of jet-powered large-scale structures is very slim for BHXRBs, mainly as a result of the jets propagating though under-dense environments compared to AGN (see Heinz 2002). Albeit the small number statistics, these large scale structures point 
towards the jets carrying away a substantial fraction of the overall accretion energy budget (Heinz \& Grimm 2005; Gallo et al. 2005; Fender, Maccarone \& van Kesteren 2005; Körding, Jester \& Fender 2008; Heinz, Merloni \& Schwab 2007, King et al. 2103b).

More recently, much excitement has grown around the claim of a positive correlation - consistent with the BZ-predicted scaling - between spin parameter and jet power for transient jets (Narayan \& McClintock 2012; Steiner, McClintock \& Narayan 2013). In these works, the mass-scaled peak radio luminosity of the giant radio flare that is often observed during hard-to-soft state transitions is taken as proxy for jet power (while the spin measurements rely on fitting the broadband $\mathrm{X}$-ray spectrum in pure thermal states). In a subsequent work, Russell, Gallo \& Fender (2013) argued against there being a significant correlation. While the controversy is still open, much of the uncertainty depends on the relative scarcity of observations of Eddington-limited BHXRBs, which are supposed to act as "standard candles" (cf. Steiner et al. 2013). The debate will likely settle over the next few years, as more and better data become available; in the meantime, the number of possible exceptions to the $P_{\mathrm{j}} \propto a^{2}$ scaling relation for transient jets may be growing; the extragalactic BHXRB in M31 (Middleton, Miller-Jones \& Fender 2014), and the Galactic system 4U 1630-472 (King et al. 2014) seem to display too high/low (respectively) flare radio luminosity for their reported spin values (unless exceptionally high values of jet boosting/de-boosting factor are at play). For the "microquasar" in M31 (Middleton et al. 2013), however, the significant uncertainty on the system inclination also allows for a reconciliation with the best-fit scaling relation reported by Steiner et al. (2013). For 4 U 1630-472, the high spin parameter reported by King et al. (2014) relies on fitting the X-ray reflection spectrum rather than thermal continuum spectrum. While the number of discrepancies between the two spin-measuring methods is reported to be diminishing (see discussions in Miller et al. 2009; Fabian et al. 2012), the consensus is that any convincing relation between jet power and spin parameter ought to be verified by both (or, at least, that either spin-fitting method leading to the existence of a positive relation with jet power does not constitute a legitimate argument for invalidating the other).

An entirely new approach to measuring black hole spin has been recently devised by Motta et al. (2014a,b); rather than fitting X-ray energy spectra, this technique relies on the relativistic precession model quasi-period oscillations (QPOs) in the power density spectra of BHXRBs (Stella \& Vietri 1999). This method has been successfully applied to the BHXRB GRO J1655-40 (Motta et al. 2014a) and XTE J1550-564 (Motta et al. 2014b). In the former case, the recovered black hole mass value matches the known system's mass function, while the spin value is not consistent with X-ray spectroscopy. In the latter, the spin value estimated from the relativistic precession model is consistent with X-ray spectroscopy (while the known black hole mass was used to solve for spin). Going forward, this could prove an exceptionally powerful method to infer black hole spin and mass values, in that it is not affected by many of the complexities of radiative models. 
3.2 Jet power and spectral breaks

As briefly discussed in the previous section, the location where the optically thick (partially self-absorbed) synchrotron spectrum breaks and becomes optically thin also determines the peak of the jet flux density. Standard synchrotron theory predicts this jet break frequency $\left(\nu_{b}\right)$ to scale with the mass accretion rate and black hole mass (Heinz \& Sunyaev 2003; Markoff et al. 2001, 2003, 2005). For BHXRBs, assuming that the magnetic field and jet acceleration region size do not vary dramatically, a positive relation should exist between $\nu_{b}$ and mass accretion rate. In order to test for such a correlation (in the form of $\nu_{b} \propto L_{\mathrm{X}}{ }^{1 / 3}$ ) Russell et al. (2013a) performed a comprehensive literature search for nearly simultaneous multi-wavelength (including radio, IR and optical) data of BHXRBs in quiescent and hard states. This work represents the most accurate and complete study on BHXBR jet spectral breaks to date, with 12 BHRXBs having constraints on $\nu_{b}$. No global relation was found with $L_{\mathrm{X}}$, from $10^{-8}$ up to the Eddington limit.

Prior to this work, a shifting jet spectral break frequency (that is, within the duration of a single outburst) had been observed for one BHXRB only; GX339-4 (Gandhi et al. 2011; Corbel et al. 2013). Russell et al. (2013a) reported on the same behavior for XTE J1118+480, whose jet break frequency varied by more than one order of magnitude while the X-ray luminosity change was negligible. More recently, high cadence, multi-wavelength monitoring of the 2011 outburst of MAXI J1836-194 (Russell et al. 2013b), showed even more severe discrepancies between the data and the expected scaling between $\nu_{b}$ and $L_{\mathrm{X}}$; during the early days of the outburst, $\nu_{b}$ for MAXI J1836-194 moved almost perpendicular to theoretical line (see Fig 4), strongly indicating a much higher degree of complexity in the processes that regulate the jet special energy distribution, particularly during the initial phases of the compact jet recovery.

A second, major finding of this multi-wavelength campaign was the observational inference, for the first time, of the high energy synchrotron cooling break: $3.2 \times 10^{14} \lesssim \nu_{c} \lesssim 4.5 \times 10^{14} \mathrm{~Hz}$. Though the cooling break was not directly visible in the broad-band spectral energy distribution data (see figure 7 of Russell et al. 2013b for a zoom into the mid-IR to X-ray spectrum of MAXI J1836-194 as measured on 2011 Sept 03, along with the inset showing the Ag 31 and Sept 01 VLT spectrum), the presence of a high energy break in the above frequency range is necessary in order to reconcile the near-IR synchrotron power-law spectrum with the strength of the Balmer absorption lines seen in the nearly simultaneous (within a day) VLT optical spectrum ${ }^{1}$. It is important to stress that, for X-ray binaries, the cooling break is expected to shift from from the ultraviolet to the $\mathrm{X}$-ray band (above $10 \mathrm{keV}$ ) as $L_{\mathrm{X}}$ increases from quiescence up to bright, hard X-ray states (Pe' er \& Markoff 2012), while the reported value for MAXI J1836-194 falls into the optical band. This has been interpreted by Russell and collaborators as evidence for the jet "already evolving early in the outburst, at the same time as the system is brightening and the disc begins filling in".

1 As discussed by Russell et al. (2013b), strong Balmer absorption lines (with relative depths as high as 20 per cent of the continuum flux) are inconsistent with a synchrotron-dominated continuum; their presence demands an optically thick disc that, at the time of the observations, was contributing to about 50 per cent of the optical emission. 


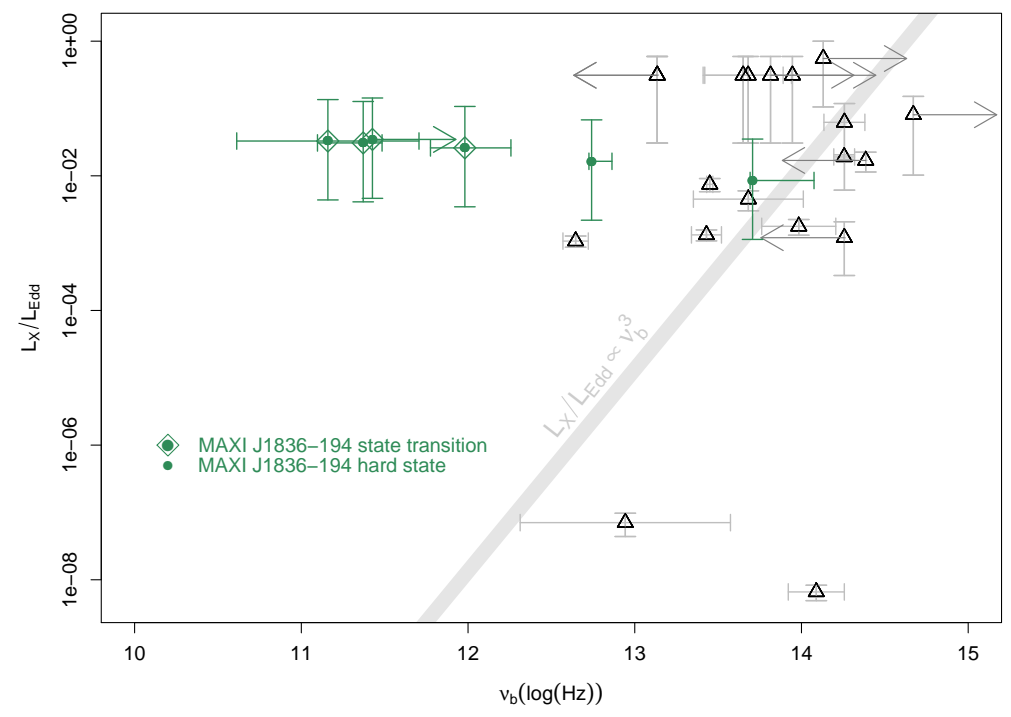

Fig. 4 Inferred values of jet spectral break frequencies as a function of Eddington scaled luminosity for a sample of 12 BHXRBs (Russell et al. 2013a,b). The evolution of MAXI J1836194, highlighted in green, is in particularly stark contrast with the simple relation expected by basic synchrotron theory plus accretion scaling arguments. Data courtesy of Dave Russell and Tom Russell.

The works discussed above serve to illustrate how high cadence, simultaneous, multi-wavelength monitoring of BHXBRs in outburst - particularly when the more traditional radio/X-ray approach is integrated with sensitive spectroscopy at IR and optical frequencies - hold the key to making further progress in this field.

A new avenue is also close to opening up for the study of X-ray binary outbursts: radio selected sources from wide-field searches. By the end of the current decade (i.e. 2020) the first phase of the SKA will have been constructed, alongside/incorporating the MeerKAT and ASKAP telescopes. For the first time large fractions of the sky (of order unity steradians) will be surveyed regularly (daily) and we should begin to find such events first by their radio emission. The mid-frequency component of the first phase of the SKA, 'SKA 1 Mid', should be able to easily detect most galactic XRBs during the hard state rising phase, providing plenty of alert for new outbursts, as well as tracking sources all the way to quiescence, and monitoring XRBs in other galaxies (Fender 2004; see www.skatelescope.org for more information on the SKA project).

\subsection{Baryons?}

One of the most important uncertainties in the study of relativistic jets ever since their discovery has been the question of their composition. In nearly all cases 
the radiation from jets is synchrotron emission, which only requires the presence of leptons, leaving us little the wiser as to whether the jets are primarily electron:proton or electron:positron in composition. The only exception to this case is SS433, which is itself an highly unusual system which is hard to fit into existing classification schemes, and in which the jet may become baryon-loaded after launch by interactions with a massive and dense environment (see Begelman, King \& Pringle 2006 and references therein).

In the past year, Diaz-Trigo et al. (2013) have reported the first evidence for strong atomic emission lines associated with relativistic jets in a (low-mass) black hole X-ray binary, 4U 1630-47. They report evidence for Doppler-shifted emission lines originating in gas moving at $\sim 0.7 c$, at the same time as optically thin radio emission is observed. This is a very exciting discovery, although it seems unlikely this can be ubiquitous for all strong-jet states and it may be only associated with certain rare phases of the outburst cycle (Neilsen et al. 2014). Regardless of the duration of the active baryon-loaded jet phase, its presence gives us very important new clues into the power of the jets and their role in the mass flow close to the black hole.

\section{Summary}

In this brief review we've attempted to provide a picture of the current empirical understanding of the coupling between accretion and outflow in stellar mass black holes. A key development in the past few years have been a clearer picture of the role of baryons in outflows, both in the systematic coupling of winds to accretion states reported in Ponti et al. (2012), and the recent, very exciting, discovery of relativistically moving baryons in a low-mass X-ray binary (Diaz-Trigo et al. 2013). Understanding these phenomena more deeply should allow us to really being to understand the flow of mass and energy near accreting black holes in different accretion states. The investigation of whether or not black hole spin really powers relativistic jets (part or all of the time) continues, and the results remain tantalising but unclear (to us). Without doubt this is one of the most important avenues of research in high-energy astrophysics research right now. What drives the 'radio loud' and 'radio quiet' branches in the hard X-ray states is a mystery (although its not spin!), and whether or not everything (anything?) we learn from black hole $\mathrm{X}$-ray binaries is really applicable to AGN remains to be proven. Much, much, more remains to be done, observationally and theoretically, and this will be in part driven by new facilties such as the SKA, and by renewed interests in the origin of X-ray binary states and accretion cycles.

Acknowledgements RPF would like to acknowledge an almost uncountable number of conversations with many collaborators over the past two decades. During this particular writing period he has learnt from Teo Munoz-Darias, Gabriele Ponti, Mickael Coriat, Dan Plant and Joey Neilsen. EG would like to thank Stephane Corbel for sharing his radio and X-ray luminosity data set, Dave and Tom Russell for sharing their data on spectral break frequencies.

\section{References}

1. Begelman M. C., King A. R., Pringle J. E., 2006, MNRAS, 370, 399 
2. Begelman M. C. \& Armitage P. J., 2014, MNRAS, 782, L18

3. Belloni T. M., Motta S. E., Munoz-Darias T., BASI, 2011, 39, 409

4. Blandford R. D., Znajek R. L., 1977, MNRAS, 179, 433

5. Blandford R. D., Payne D. G., 1982, MNRAS, 199, 883

6. Bradley C. K. et al., 2007, ApJ, 667, 427

7. Brocksopp C. et al., 2010, MNRAS, 404, 908

8. Casella P. G. et al., 2010, MNRAS, 404, L21

9. Calvelo D. E. et al., 2010, MNRAS, 409, 839

10. Chaty S. et al., 2003, MNRAS, 346, 689

11. Corbel S. et al., 2003, A\&A, 400, 1007

12. Corbel S., Körding E., Kaaret P., 2008, MNRAS, 389, 1697

13. Corbel S. et al., 2013, MNRAS, 431, L107

14. Coriat M. et al., 2011, MNRAS, 414, 677

15. Coriat M. , Fender R. P. \& Dubus G., 2012, MNRAS, 424, 1991

16. Done C., Gierlinski M., Kubota A., 2007, A\&ARv, 15, 1

17. Dunn R. J. H. et al., 2010, MNRAS, 403, 61

18. Falcke H., Körding E. \& Markoff S., 2004, A\&A, 414, 895

19. Fender R., 2004, New Astronomy Reviews, 48, 1399

20. Fender R. P., Gallo E. \& Jonker P. G., 2003, MNRAS, 343, L99

21. Fender R. P., Belloni T. \& Gallo E., 2004, MNRAS, 355, 1105

22. Fender R. P., Maccarone T. J. \& van Kesteren Z., 2005, MNRAS, 360, 1085

23. Fender R. P., 2006, Jets from X-Ray Binaries. Cambridge Univ. Press, Cambridge, p. 381

24. Fender R. P., Gallo E., Russell D. M., 2010, MNRAS, 406, 1425

25. Fender R. P. \& Belloni T., 2012, Science, 337, 540

26. Fabian A. C. et al., 2012, MNRAS, 424, 217

27. Gallo E., Fender R. P. \& Pooley G. G., 2003, MNRAS, 344, 60

28. Gallo E. et al., 2005, Nature, 436, 819

29. Gallo E., Fender R. P. \& Hynes R., 2005, MNRAS, 356, 1017

30. Gallo E. et al., 2006, MNRAS, 370, 1351

31. Gallo E. et al., 2007, ApJ, 670, 600

32. Gallo E., Miller B. P. \& Fender R. P., 2012, MNRAS, 423, 590

33. Gandhi P. et al., 2011, ApJ, 740, L13

34. Heinz S., 2002, A\&A, 388, L40

35. Heinz S. \& Sunyaev R., 2003, MNRAS, 343, L59

36. Heinz S. \& Grimm H., 2005, ApJ, 633, 384

37. Heinz S., Merloni A. \& Schwab J., 2007, ApJ, 658, L9

38. Hjellming, R. M. \& Rupen M., 1995, Nature, 6531, 464

39. Hjellming, R. M., \& Han, X., 1995, Radio Properties of X-ray binaries, p. 308

40. Hynes R. et al., 2004, ApJ, 611, L125

41. Hynes R. et al., 2006, ApJ, 651, 401

42. Hynes R. et al., 2009, MNRAS, 399, 2239

43. Khandai N. et al., 2014, MNRAS submitted (arXiv:1402.0888)

44. Körding E. G., Fender R. P. \& Migliari S., 2006, MNRAS, 369, 1451

45. Körding E. G., Jester S. \& Fender R. P., 2006, MNRAS, 372, 1366

46. Körding E. G. et al., 2007, MNRAS, 380, 301

47. Körding E. G., Jester S. \& Fender R. P., 2008a, MNRAS, 383, 277

48. Körding E.G. et al. 2008b Science, 320, 1318

49. King A. L. et al., 2013a, ApJ, 771, 84, 12

50. King A. L. et al., 2013b, ApJ, 762, 18

51. King A. L. et al., 2014, ApJ, 784, L2, 6

52. Kormendy J. \& Ho, L. 2013, ARAA, 51, 511

53. Jamil, O.; Fender, R. P.; Kaiser, C. R., 2010, MNRAS, 401, 394

54. Joinet, A.; Kalemci, E.; Senziani, F., 2008, ApJ, 679, 655

55. Jonker P. G. et al., 2010, MNRAS, 401, 1255

56. Liu B. F. et al., 2007, ApJ, 671, 695

57. Malzac J., 2013, MNRAS, 429, L20

58. Malzac J., Merloni A. \& Fabian A. C., 2004, MNRAS, 351, 253

59. Markoff S., Falcke H. \& Fender R. P., 2001, A\&A, 372, L25

60. Markoff S., et al., 2003, A\&A, 397, 645

61. Markoff S., Nowak M. \& Wilms J., 2005, ApJ, 635, 1203

62. Markoff S. \& Nowak M. , 2007, ApJ, 609, 972 
63. Martí J., 2005, MmSAI, 76, 592

64. Miller, J. M., Homan, J., Miniutti, G., 2006, ApJ, 652, L113

65. Miller, J. M., 2007, ARAA, 45, 441

66. McClintock J. E. \& Remillard R. A., 2006, Black Hole Binaries. Cambridge Univ. Press, Cambridge, p. 157

67. McClintock J. E., Narayan R., \& Steiner, J. 2013, Space Science Reviews (arXiv:1303.1583)

68. McHardy I. M. et al., 2006, Nature, 444, 730

69. Merloni A., Heinz S. \& Di Matteo T., 2003, MNRAS, 345, 1057

70. Meyer F., Liu B. F. \& Meyer-Hofmeister E., 2007, A\&A, 463, 1

71. Meyer-Hofmeister E. \& Meyer F., 2014, A\&A, 562, 142

72. Middleton, Miller-Jones J. C. A. \& Fender R. P., 2014, MNRAS, 439, 1740

73. Miller J. M., 2006, Nature, 441, 953

74. Miller J. M., 2007, ARAA, 45, 441

75. Miller J. M., 2008, ApJ, 680, 1359

76. Miller J. M. et al., 2009, ApJ, 697, 900

77. Miller J. M. et al., 2012, ApJ, 759, L6

78. Miller-Jones J. C. A. et al., 2011, ApJ, 739, L18

79. Miller-Jones J. C. A. et al., 2012, MNRAS, 421, 468

80. Mirabel I. F. \& Rodríguez L. F., 1994, Nature, 371, 46

81. Mirabel I. F. et al., 1998, A\&A, 330, L9

82. Mirabel I. F. \& Rodríiguez L. F., 1999, ARAA, 37, 409

83. Motta, S. E.; Belloni, T.; Homan, J., 2009, MNRAS, 400, 1603

84. Motta S. E. et al., 2014a, MNRAS, 437, 2554

85. Motta S. E. et al., 2014b, MNRAS, 439, L65

86. Munoz-Darias T., Motta S., Belloni T., 2011, MNRAS, 410, 679

87. Narayan R., McClintock J. E., 2012, MNRAS, 419, L69

88. Neilsen J. \& Lee J.C., 2009, Nature, 458, 481

89. Neilsen J. et al. 2014, ApJL, 784, L5

90. Petrucci P.O. et al. 2008, MNRAS, 385, L88

91. Pe' er A. \& Markoff S., 2012, ApJ, 753, 177

92. Plant D.S.et al. 2014, MNRAS, submitted

93. Ponti G. et al., 2012, MNRAS, 422, 11

94. Remillard R. \& McClintock J.E., 2006, ARA\&A, 44, 49

95. Russell D. M. et al. 2003, MNRAS, 371, 13

96. Russell D. M. et al. 2007, MNRAS, 379, 1401

97. Russell D. M. et al. 2011a, ApJ, 739, L19

98. Russell D. M. et al. 2011b, MNRAS, 416, 2311

99. Russell D. M., Gallo E. \& Fender R., 2013, MNRAS, 431, 405

100. Russell D. M. et al., 2013a, ApJ, 768, L35

101. Russell D.M., Shahbaz T., 2014, MNRAS, 438, 2083

102. Russell T. D. et al. 2013b, MNRAS in press (2014MNRAS.tmp..180R)

103. Reis R. C., Fabian A. C. \& Miller J. M., 2010, MNRAS, 402, 836

104. Reynolds C. S., 2013, Space Science Reviews (arXiv:1302.3260)

105. Reynolds M. T. \& Miller J. M., 2013, ApJ, 769, 16

106. Soleri P., Belloni T., CAsella P., 2008, MNRAS, 383, 1089

107. Soleri P. \& Fender R., 2011, MNRAS, 413, 2268

108. Steiner J. F., McClintock J. E. \& Narayan R., 2013, ApJ, 762, 104

109. Stella L. \& Vietri M., 1999, Phys. Rev. Lett., 82, 17

110. Tchekhovskoy A. et al., 2010, ApJ, 711, 50

111. van der Laan H., 1966, Nature, 211, 1131 